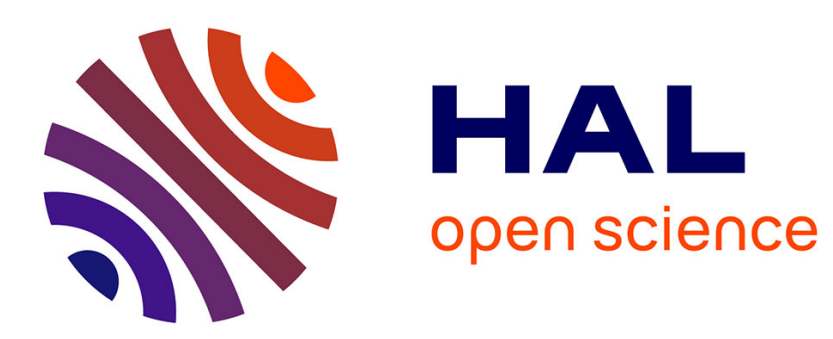

\title{
Dispositif de mesure du dichroisme linéaire dans l'ultraviolet à vide
}

\author{
H. Damany, J.A. Schellman, V. Chandrasekharan
}

\section{To cite this version:}

H. Damany, J.A. Schellman, V. Chandrasekharan. Dispositif de mesure du dichroisme linéaire dans l'ultraviolet à vide. Revue de Physique Appliquée, 1970, 5 (2), pp.259-262. 10.1051/rphysap:0197000502025900 . jpa-00243385

\section{HAL Id: jpa-00243385 https://hal.science/jpa-00243385}

Submitted on 1 Jan 1970

HAL is a multi-disciplinary open access archive for the deposit and dissemination of scientific research documents, whether they are published or not. The documents may come from teaching and research institutions in France or abroad, or from public or private research centers.
L'archive ouverte pluridisciplinaire HAL, est destinée au dépôt et à la diffusion de documents scientifiques de niveau recherche, publiés ou non, émanant des établissements d'enseignement et de recherche français ou étrangers, des laboratoires publics ou privés. 


\title{
DISPOSITIF DE MESURE DU DICHROISME LINÉAIRE DANS L'ULTRAVIOLET A VIDE
}

\author{
H. DAMANY, J. A. SCHELLMAN $\left({ }^{1}\right)$ et V. CHANDRASEKHARAN \\ Laboratoire des Hautes Pressions, C. N. R. S., 92, Bellevue
}

(Reçu le 6 octobre 1969)

\begin{abstract}
Résumé. - Description d'un dispositif de mesure du dichroïsme linéaire dans l'ultraviolet à vide par modulation de la polarisation au moyen d'un prisme de Rochon tournant. La sensibilité absolue est contrôlée par la mesure de la transmission pseudo-dichroïque d'une lame de quartz fondu inclinée. Le signal détectable minimal est $10^{-3}$. Le spectre dichroïque d'un biopolymère orienté est présenté comme exemple.
\end{abstract}

\begin{abstract}
An apparatus for measuring linear dichroism in the vacuum ultraviolet by modulating the polarisation of light with a rotating quartz Rochon is described. The absolute sensitivity is tested by the measurement of the pseudo-dichroic transmission of an inclined fused quartz plate. The minimum detectable signal is $10^{-3}$. The dichroic spectrum of an oriented biopolymer is presented as an example.
\end{abstract}

1. Introduction. - L'intérêt que présente en biophysique moléculaire [1] l'étude du dichroïsme linéaire (DL) dans l'ultraviolet lointain ne peut être que stimulé par l'avènement de techniques de mesures rapides; et il apparaît souhaitable, en outre, d'étendre le domaine spectral aux longueurs d'onde inférieures à $2000 \AA$. Une méthode récente [2] consiste à placer un polariseur et une lame multi-onde sur le trajet du faisceau de mesure d'un spectrophotomètre à deux faisceaux ; on obtient ainsi, en alternance, à des intervalles de longueur d'onde plus ou moins grands, des polarisations orthogonales. Cette méthode présente l'avantage d'une adaptation rapide mais, de par son principe, présente certains inconvénients : la mesure du DL ne peut s'opérer qu'aux longueurs d'onde fixes où la lame présente un retard de $\lambda / 2$; en outre des corrections sont nécessaires qui dépendent du profil instrumental de l'appareil dispersif et qui limitent la résolution des mesures du DL ; enfin, vers les courtes longueurs d'onde, intervient la dispersion de la biréfringence de la lame.

Le principe de l'appareil que nous décrivons dans cet article a été suggéré par l'un des auteurs $\left({ }^{2}\right)$ et envisagé à l'origine, non seulement pour des mesures de DL, mais également pour des mesures de dichroïsme circulaire. Il consiste à moduler la polarisation par rotation continue du polariseur à une fréquence

(1) Actuellement : Department of Chemistry, University of Oregon, Eugene, Oregon (U. S. A.).

(2) J. A. Schellman. $f=\omega / 2 \pi$; le D. L. d'un échantillon se traduit par la superposition au signal continu d'un signal alternatif de fréquence $2 \mathrm{f}$ qu'il est aisé d'amplifier sélectivement et de détecter. Nous avons donné par ailleurs [3] en même temps que les résultats de mesures jusqu'à $1500 \AA$ du DL de couches orientées de biopolymères, une brève description de la méthode et de ses performances. Nous en présentons ici les détails, l'évaluation de sa précision et de ses limites ainsi que certaines applications autres que la mesure du DL.

2. Description du dispositif expérimental. - $\mathrm{La}$ figure 1 est un schéma de l'appareillage. En suivant la propagation de la lumière, on trouve une lampe à

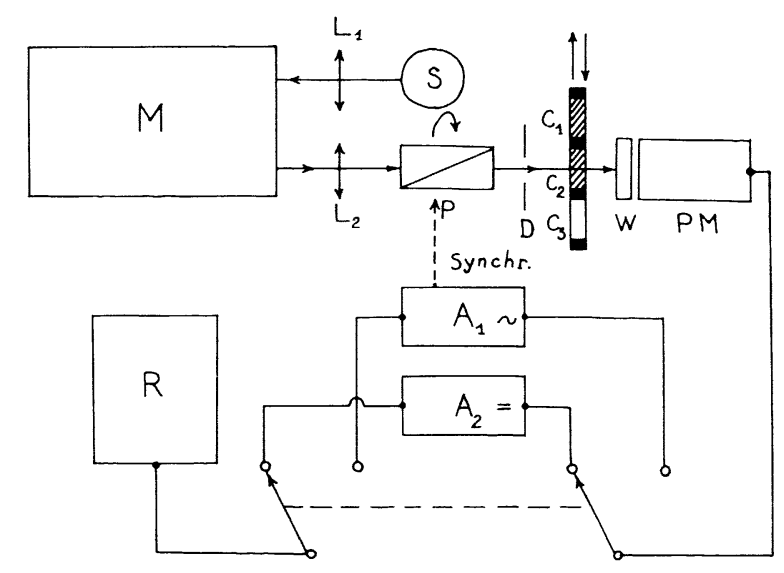

FIG. 1. - Schéma de l'appareillage. 
hydrogène $\mathrm{S}$, une lentille $\mathrm{L}_{1}$ assurant une meilleure collection de la lumière, un monochromateur à vide à réseau en incidence normale, une lentille $L_{2}$ donnant un faisceau quasi parallèle, un prisme de Rochon $\mathrm{R}$ en quartz synthétique, un diaphragme $\mathrm{D}$ éliminant le faisceau dévié, l'échantillon à mesurer et enfin le récepteur comportant une fenêtre recouverte de salicylate de sodium et un photomultiplicateur; sont associés au photomultiplicateur un amplificateur de courant alternatif à détection synchrone $\mathrm{A}_{1}$, un amplificateur de courant continu $\mathrm{A}_{2}$ et enfin un enregistreur. Une commutation mécanique permet ainsi d'enregistrer soit le signal continu du photomultiplicateur, soit un signal alternatif. Le prisme de Rochon est monté à l'intérieur d'une poulie et tourne à $900 \mathrm{t} / \mathrm{mn}$, le couplage mécanique avec le moteur synchrone, qui lui est à l'extérieur de l'enceinte à vide, est réalisé par un passage tournant étanche, un ensemble de poulies et une courroie. La fréquence de modulation de la polarisation est ainsi de $30 \mathrm{~Hz}$. Le signal de référence pour la détection synchrone était fourni, dans une première version, par un contact magnétique ; celui-ci fut avantageusement remplacé par la suite par un phototransistor associé à un disque tournant ajouré et une ampoule à filament; ce dernier dispositif donne des impulsions parfaitement reproductibles dans le temps. Un porte-échantillon à trois positions permet de faire les mesures consécutivement sur l'échantillon, sur une fenêtre de référence et enfin sur le faisceau direct pour en contrôler l'intensité et le pourcentage de modulation parasite.

Si $I_{0}$ est l'intensité du faisceau à la sortie du monochromateur, cette intensité devient après passage à travers le polariseur et l'échantillon dichroïque

$$
I=K \frac{I_{0}}{4}\left[\sum E+\Delta E \cos 2 \Omega t\right]
$$

avec

$$
{ }_{\Delta}^{\Sigma} \mid E=\mathrm{e}^{-4 \pi \kappa \perp 1 / \lambda} \pm \mathrm{e}^{-4 \pi \kappa / /^{1 / \lambda}}
$$

$\kappa_{\perp}$ et $\kappa_{\|}$étant les indices d'extinction principaux de la substance dichroïque; $K$ est un paramètre qui ne dépend que de la longueur d'onde et qui représente les différentes pertes d'intensité dans le polariseur ou la lame support de l'échantillon.

On mesure alors consécutivement, à chaque $\lambda$, un signal continu $K S\left(I_{0} / 4\right) \sum E$ et un signal alternatif $K S\left(I_{0} / 4\right) \Delta E$ et on obtient en définitive

1) la transmission de l'échantillon

$$
T_{\mathrm{m}}=\sum E / 2=\left(T_{\perp}+T_{\|}\right) / 2,
$$

2) le degré de polarisation après traversée de l'échantillon

$$
P=\left(T_{\perp}-T_{\|}\right) /\left(2 T_{\mathrm{m}}\right)=\Delta E / \sum E .
$$

La mesure de $P$ permet ainsi d'atteindre le DL, qui est défini classiquement par $\Delta \kappa=\kappa_{\perp}-\kappa_{\|} \cdot P$ peut être tracé automatiquement si on utilise une électronique de rapport, ce que nous envisageons de faire dans le futur.

3. Discussion et performance. - La sensibilité de la méthode dépend du degré de polarisation parasite $P_{0}$ que l'on observe en l'absence d'échantillon. Sans précautions particulières, $P_{0}$ peut être important. On le réduit dans des proportions considérables par un alignement rigoureux de tous les éléments optiques et, en particulier, du prisme de Rochon ; ce dernier doit non seulement être centré sur le faisceau, mais, en outre, ses faces d'entrée et de sortie doivent être parfaitement parallèles; cette dernière condition est réalisée par polissage des faces après contact optique des deux parties du prisme (parallélisme à mieux que $20^{\prime \prime}$ ). Avec ces précautions, nous obtenons un $P_{0}$ inférieur à $0,1 \%$ - pour une constante de temps de $1 \mathrm{~s}$ de l'amplificateur synchrone - dans toute la région 1 670-3000 Å qui correspond au continu de l'hydrogène. $P_{0}$ est plus important dans la région du spectre de raies; il atteint $0,5 \%$ à $1608 \AA$ et environ $1 \%$ à $1490 \AA$, c'est-à-dire vers la limite de transparence du Rochon de quartz. L'origine de cette modulation résiduelle n'est pas évidente mais on peut l'attribuer à différentes causes : 1) des réflexions multiples entre la lentille de focalisation $\mathrm{L}_{2}$ et la face d'entrée du Rochon : on vérifie que la suppression de la lentille entraîne une petite diminution de $P_{0} ; 2$ ) des phénomènes au niveau de la surface de contact des deux parties du prisme de Rochon, par exemple une transmission non uniforme ; 3) enfin, des vibrations mécaniques des éléments en rotation.

Les valeurs précédentes de $P_{0}$ donnent l'ordre de grandeur des plus faibles signaux dichroïques mesurables par la présente méthode.

Notons enfin que dans les conditions où nous opérons, aucun dépolariseur n'est nécessaire. En effet, on ne détecte aucune polarisation partielle due au monochromateur, ce qui s'explique par la grande activité optique du quartz dans la direction de l'axe optique. Or, dans le premier des deux prismes constituant le Rochon, la lumière se propage suivant l'axe optique et subit une rotation considérable du plan de polarisation $\left(270^{\circ} / \mathrm{mm}\right.$ à $\left.\lambda=2073 \AA\right)$; le trajet parcouru par les différents rayons variant de 0 à plusieurs millimètres, la lumière est donc totalement dépolarisée à l'interface des deux prismes. On le vérifie aisément en plaçant un polariseur parfait avant le Rochon tournant : aucun signal alternatif n'est observé. En outre, à la sortie, la lumière de fluorescence du salicylate étant totalement dépolarisée, aucun dépolarisateur n'est nécessaire avant le photomultiplicateur.

4. Etalonnage. - Pour effectuer l'étalonnage de l'appareil, on s'est servi d'une lame mince de calcite, d'épaisseur $50 \mu$, taillée parallèlement à l'axe optique ; une telle lame constitue un polariseur parfait dans la région $1670<\lambda<1850 \AA$ [4]. Mise à la place de 
l'échantillon, elle permet, dans le domaine spectral indiqué, d'ajuster le niveau du signal alternatif détecté de façon à obtenir $P=100 \%$.

5. Essais. - Un premier type d'essai a consisté à utiliser une lame transparente inclinée comme substance pseudo-dichroïque. Elle agit comme polariseur partiel et ses transmissions principales sont :

$$
\begin{aligned}
T_{\perp} & =\left[\frac{\sin ^{2}\left(\theta_{i}+\theta_{r}\right)}{\sin ^{2}\left(\theta_{i}+\theta_{r}\right)} \frac{\sin ^{2}\left(\theta_{i}-\theta_{r}\right)}{2}\right]^{2} \\
T_{\|} & =\left[\frac{\operatorname{tg}^{2}\left(\theta_{i}+\theta_{r}\right)-\operatorname{tg}^{2}\left(\theta_{i}-\theta_{r}\right)}{\operatorname{tg}^{2}\left(\theta_{i}+\theta_{r}\right)}\right]^{2}
\end{aligned}
$$

$\theta_{i}$ et $\theta_{r}$ sont respectivement les angles d'incidence et de réfraction, et sont liés par la relation $\sin \theta_{i}=n \sin \theta_{r}$, $n$ étant l'indice du matériau. Le degré de polarisation partielle à la sortie de la lame est

$$
\begin{aligned}
P & =\left(T_{\|}-T_{\perp}\right) /\left(T_{\|}+T_{\perp}\right) \\
& =\left[1-\cos ^{4}\left(\theta_{i}-\theta_{r}\right)\right] /\left[1+\cos ^{4}\left(\theta_{i}-\theta_{r}\right)\right] .
\end{aligned}
$$

Dans le cas $n>1$, on en déduit

$$
n=\sin \theta_{i} /\left(Q \sin \theta_{i}-\sqrt{1-Q^{2}} \cos \theta_{i}\right)
$$

avec

$$
Q=\cos \left(\theta_{i}-\theta_{r}\right)=[(1-P) /(1+P)]^{1 / 4} .
$$

Ces équations ne sont valables que si aucun des rayons subissant des réflexions multiples n'atteint le détecteur.

Les relations (8) et (9) permettent de calculer $n$ à partir des mesures de $P$ lorsque l'on connaît $\theta_{i}$. Mais, afin de choisir la valeur optimale de $\theta_{i}$, on a calculé, à l'aide d'une machine électronique, les valeurs de $P$ en fonction de $n$ et $\theta_{i}, n$ variant de 1,4 à 2,0 avec un pas de 0,01 et $\theta_{i}$ de 0 à $85^{\circ}$ avec un pas de $5^{\circ} . \mathrm{d} P / \mathrm{d} n$ est représentatif de la sensibilité de la méthode et l'on peut facilement montrer que $P$ et $\mathrm{d} P / \mathrm{d} n$ sont tous les deux des fonctions monotones croissantes de $\theta_{i}$. Ce sont donc les dimensions latérales de la lame qui

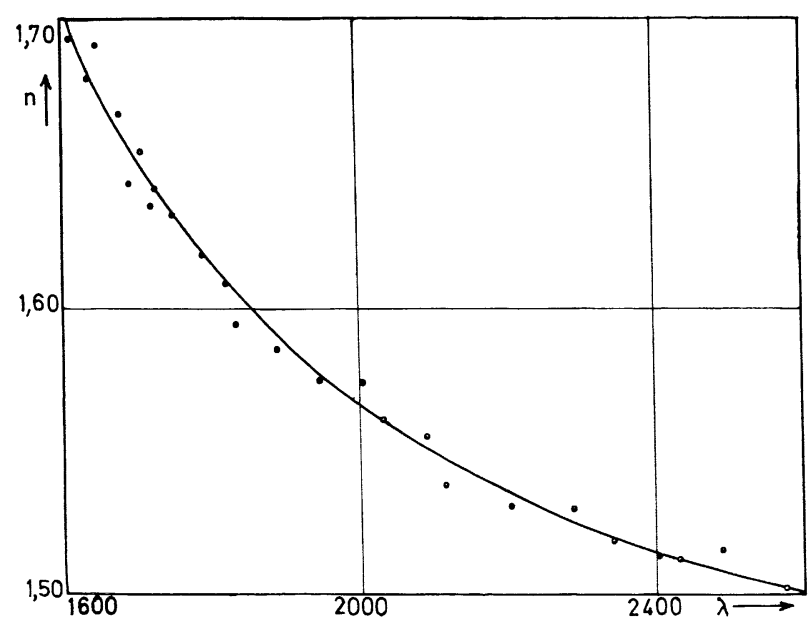

FIG. 2. - Dispersion de l'indice de réfraction du quartz fondu. imposent une limite de fait à l'angle $\theta_{i}$. Nous avons choisi comme compromis un angle de $45^{\circ}$. Avec cet angle, $P=0,13$ et $\mathrm{d} P / \mathrm{d} n=0,2$ pour $n=1,7$, de sorte que l'on doit pouvoir détecter facilement une variation d'indice de réfraction de l'ordre de $0,005 \mathrm{si}$ l'instrument est à même de détecter un $\Delta P$ de 0,001 .

Pour un premier essai, nous avons utilisé une lame de quartz fondu (de diamètre $30 \mathrm{~mm}$ ) dont la transparence s'étend jusqu'à 1570 grâce à la qualité du poli. Sur la figure 2 , nous donnons les résultats des mesures de dispersion et indice de ce matériau; la précision absolue sur l'indice est $\pm 0,01$ et correspond assez bien à l'incertitude sur $P$ due à $P_{0}$.

Un second essai a consisté à étudier l'absorption dichroïque d'une couche mince orientée de poly- $\gamma$ -éthyl-glutamate déposée sur une lame de fluorine. La figure 3 montre la variation avec la largeur d'onde

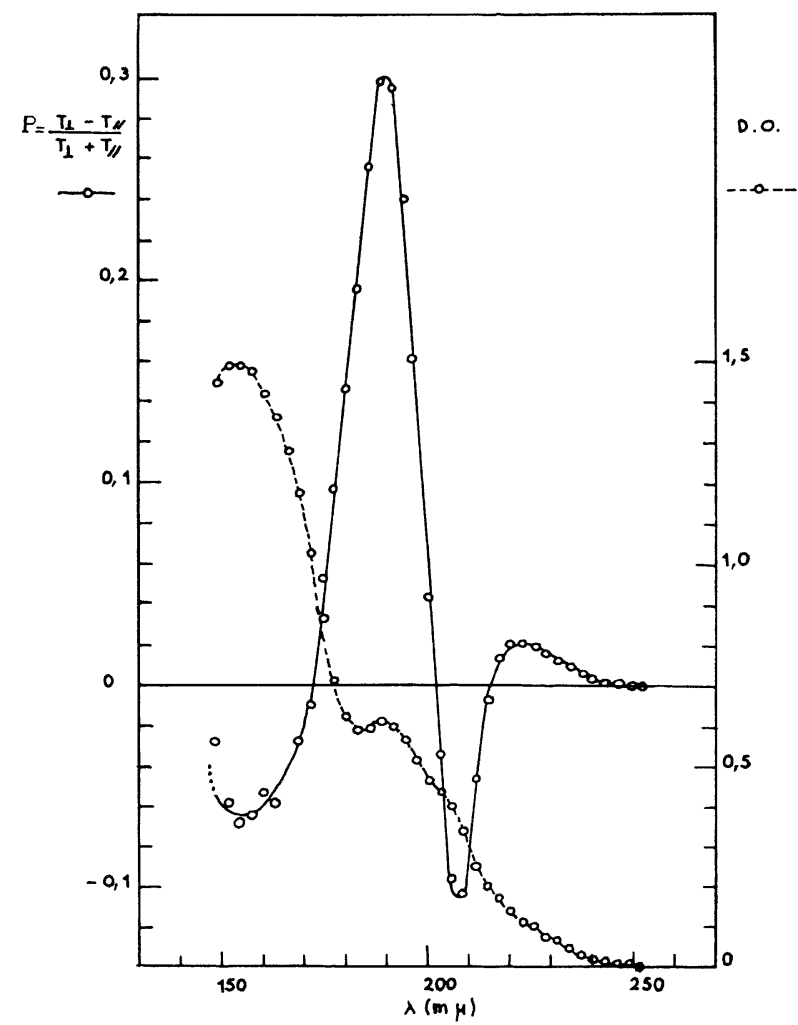

FIG. 3. - Densité optique et spectre dichrö̈que du poly-y-éthyl L-glutamate orienté.

de la densité optique de ce matériau (courbe en pointillés), d'une part, et de la quantité $P$, d'autre part (courbe en trait continu) qui constitue une mesure du dichroïsme linéaire de l'échantillon. Notons les structures qui apparaissent nettement sur la courbe $P$ et dont certaines se traduisent par un changement de signe (la discussion théorique en a été présentée par ailleurs [3]). Cet exemple illustre bien les possibilités de la méthode.

La limite actuelle en longueur d'onde est imposée par la transparence du quartz du Rochon; avec un rochon de fluorure de magnésium [5], il'serait possible 
d'étendre les mesures jusqu'à $1350 \AA$, mais il faudrait lui adjoindre nécessairement un dépolariseur pour éliminer la polarisation partielle introduite par le réseau. Des améliorations supplémentaires portant sur l'alignement optique des pièces tournantes et sur le signal de référence permettraient sans doute aussi d'augmenter la sensibilité au-delà de la limite actuelle en diminuant $P_{0}\left(P_{0} \sim 0,05 \%\right)$.

\section{Bibliographie}

[1] Gratzer (W. B.), HolzWarth (G. M.), Proc. Nat. Acad. Sci., 1961, 47, 1785.

[2] Jaffe (J. H.) et JAFFe (H.), Rev. Sci. Inst., 1967, 38, 935.

[3] Brahms (J.), Pilet (J.), Damany (H.) and Chandra.
SeKharan (V.), Proc. Nat. Acad. Sci.,, 1968, 60, 1130.

[4] Schellman (J. A.), Chandrasekharan (V.) et Damany (H.), C. R. Acad. Sci., Paris, 1965, 260, 117.

[5] Steimetz (D. L.), Phillips (W. G.), Wirick (M.) and Forbes (F. F.), Appl. Opt., 1967, 6, 1001. 